\title{
Computing the preconditioner for the Schur complement
}

\author{
K. Moriya* $\quad$ T. Nodera ${ }^{\dagger}$ \\ (Received 27 October 2004; revised 11 March 2005)
}

\begin{abstract}
The Newton scheme is used to construct an approximate inverse preconditioner for the Schur complement. However, this scheme is very expensive because of the computation cost of the matrix-matrix product. In this paper, the computation cost of the Newton scheme is reduced by implementing the preconditioner implicitly using the matrix-vector product. We also show that such an implementation is less expensive than computing the preconditioner explicitly.
\end{abstract}

\section{Contents}

\section{Introduction}

C395

*Dept. of Information and Integrated Tech., Aoyama Gakuin University, JAPAN. mailto:moriya@it.aoyama.ac.jp

†Dept. of Math., Keio University, JAPAN. mailto:nodera@math.keio.ac.jp

See http://anziamj.austms.org.au/V46/CTAC2004/Mori for this article, (c) Austral. Mathematical Soc. 2005. Published May 23, 2005. ISSN 1446-8735 
2 Composing the Schur complement $\quad$ C396

2.1 Independent set . . . . . . . . . . . . . . . . . C396

2.2 Reordering the rows and columns . . . . . . . . . C396

3 Implementation of the preconditioner $\quad$ C397 3.1 Initial guess in Newton iteration . . . . . . . . . . . C397

3.2 Newton scheme without the matrix-matrix product . . . . C398

4 Numerical experiments $\quad \mathbf{C 4 0 0}$ 4.1 First example . . . . . . . . . . . . . . . . . . C400

4.2 Second example . . . . . . . . . . . . . . . . C404

5 Concluding remarks

C407

References

C407

\section{Introduction}

We consider the problem of solving a large and sparse linear system of equations:

$$
A \boldsymbol{x}=\boldsymbol{b}, \quad A \in R^{n \times n}, \quad \boldsymbol{x}, \boldsymbol{b} \in R^{n} .
$$

In order to reduce the size of the linear system (1), we arrange a reduced linear system with a Schur complement by using an independent set. The Schur complement is composed by reordering the rows and columns of matrix $A$. We aim to apply an approximate inverse preconditioner to a reduced linear system with the Schur complement to improve the convergence rate. We are interested in the Newton scheme for computing the approximate inverse preconditioner since it is simple to implement. However, the matrix-matrix product is more difficult to compute as the preconditioner is less sparse. In this paper, we implement the Newton scheme without the matrix-matrix product. This implementation computes the preconditioner implicitly by us- 
ing the matrix-vector product. The numerical results suggest that computing the preconditioner implicitly is more effective than computing it explicitly.

This paper is organized as follows. Section 2 provides an overview of composing the Schur complement. In Section 3, we consider the Newton scheme for the approximate inverse preconditioner and implement the preconditioner implicitly. The numerical results are reported in Section 4, and the concluding remarks are presented in Section 5.

\section{Composing the Schur complement}

\subsection{Independent set}

In this subsection, we introduce the independent set $G$. The indices $s_{i}$ and $s_{j}$ are independent of each other if the entries $a_{s_{i} s_{j}}, a_{s_{j} s_{i}} \in A\left(s_{i} \neq s_{j}\right)$ are zero. All the indices belonging to $G$ must be independent of each other. Therefore, define

$$
G:=\left\{s_{1}, s_{2}, \ldots, s_{k} \mid a_{s_{i} s_{j}}=0, a_{s_{j} s_{i}}=0, \text { for all } i, j=1,2, \ldots, k\right\},
$$

where $k$ is the number of entries of $G$. The independent set $G$ is constructed by using the Greedy algorithm. For more details on the implementation of the Greedy algorithm, see [7].

\subsection{Reordering the rows and columns}

Let $G \in\left\{s_{1}, s_{2}, \ldots, s_{k}\right\}$ be the independent set selected from the row and column indices of matrix $A$. The components of the linear system (1) are rearranged into the forms

$$
P A P^{T}=\left(\begin{array}{cc}
A_{1} & A_{2} \\
A_{3} & A_{4}
\end{array}\right), \quad P \boldsymbol{x}=\left(\begin{array}{c}
\boldsymbol{x}_{1} \\
\boldsymbol{x}_{2}
\end{array}\right), \quad P \boldsymbol{b}=\left(\begin{array}{c}
\boldsymbol{b}_{1} \\
\boldsymbol{b}_{2}
\end{array}\right),
$$


where $P$ is a suitable permutation matrix. The row and column indices of sub-matrix $A_{1}$ belong to $G$. Since $A_{1}$ is usually a diagonal matrix, it is not difficult to obtain $A_{1}^{-1}$. From the reordering forms (2), the following reduced linear system of size $\tilde{n}=n-k$ is generated:

$$
C \boldsymbol{x}_{2}=\tilde{\boldsymbol{b}}
$$

where

$$
C=A_{4}-A_{3} A_{1}^{-1} A_{2}, \quad \tilde{\boldsymbol{b}}=\boldsymbol{b}_{2}-A_{3} A_{1}^{-1} \boldsymbol{b}_{1} .
$$

The coefficient matrix $C$ is the so-called Schur complement of sub-matrix $A_{1}$. The nonzero entries of matrix $C$ need not be stored since the iterative solver of the linear system requires $C \boldsymbol{v}$. Therefore, only four products $A_{1}^{-1} \boldsymbol{v}_{1}$, $A_{2} \boldsymbol{v}_{2}, A_{3} \boldsymbol{v}_{3}$ and $A_{4} \boldsymbol{v}_{4}$ have to be computed.

\section{Implementation of the preconditioner}

In this section, we use the Newton scheme to develop the approximate inverse preconditioner.

\subsection{Initial guess in Newton iteration}

Schulz [1] proposed the Newton formula

$$
N_{l+1}=\left(2 I-N_{l} C\right) N_{l},
$$

where $N_{l}$ is the preconditioner of matrix $C$ in the $l$ th Newton iteration. The convergence of the Newton scheme depends on the initial guess $N_{0}$. The preconditioner $N_{l}$ converges to matrix $C^{-1}$ if the spectral radius $\rho\left(I-N_{0} C\right)$ is less than 1.0 [8]. Ben-Israel et al. [3] proved that this preconditioner converges to $C^{-1}$ if the Newton iteration begins with

$$
N_{0}=\alpha C^{T},
$$


and the condition

$$
0<\alpha<\frac{2}{\rho\left(C C^{T}\right)}
$$

is satisfied (Householder $[2$, p.95]). Here, $\rho($.$) denotes the spectral radius of$ the matrix. Pan et al. [6] selected $\alpha=1 /\left(\|C\|_{1}\|C\|_{\infty}\right)$. This makes

$$
N_{0}=\frac{C^{T}}{\|C\|_{1}\|C\|_{\infty}},
$$

where $\|C\|_{1}=\max _{j} \sum_{i=1}^{\tilde{n}}\left|c_{i j}\right|$ and $\|C\|_{\infty}=\max _{i} \sum_{j=1}^{\tilde{n}}\left|c_{i j}\right|$. However, the problem with this selection is that all the nonzero entries of matrix $C$ are required for computing the matrix norms $\|C\|_{1}$ and $\|C\|_{\infty}$. All the nonzero entries of $C$ must be computed with the matrix-matrix product. Therefore, it is very expensive to compute the initial guess $N_{0}$ with equation (7).

On the other hand, we select the diagonal matrix

$$
N_{0}=\operatorname{diag}\left\{1 / c_{11}, 1 / c_{22}, \ldots, 1 / c_{\tilde{n} \tilde{n}}\right\}
$$

as the initial guess, where $c_{i i}$ is the $i$ th diagonal entry of matrix $C$. The advantages of this choice are that only the diagonal entries of $C$ are needed and the number of nonzero entries is limited to $\tilde{n}$. The numerical results reported in Section 4 show that $N_{l}$ performs well as the preconditioner even if the equation (8) is selected as the initial guess $N_{0}$. In general, the Newton formula (4) does not always converge to $C^{-1}$ even if the equation (8) is selected. However, we focus on reducing the computation cost of $N_{0}$. Moreover, in Section 4, we show that the selection (7) as the initial guess $N_{0}$ is more expensive than selecting (8).

\subsection{Newton scheme without the matrix-matrix product}

The difficulty in using the Newton formula (4) is that the matrix-matrix product is often very expensive to compute. In order to overcome this draw- 
back, we implement the Newton scheme without the matrix-matrix product. The main goal of this implementation is to avoid matrix-matrix products in the Newton scheme by using the matrix-vector product. The iterative solver does not need the preconditioner $N_{l}$ in explicit form; Instead, it requires $N_{l} \boldsymbol{v}$, where $\boldsymbol{v}$ is an arbitrary vector. Therefore, we compute $N_{l}$ implicitly by using $N_{l} \boldsymbol{v}$. By substituting the Newton formula (4) in the equation $\boldsymbol{w}_{l}=N_{l} \boldsymbol{v}$, we describe vectors $\boldsymbol{w}_{0}, \boldsymbol{w}_{1}, \boldsymbol{w}_{2}$ and $\boldsymbol{w}_{3}$ :

$$
\begin{aligned}
\boldsymbol{w}_{0}= & N_{0} \boldsymbol{v}, \\
\boldsymbol{w}_{1}= & \left(2 I-N_{0} C\right) N_{0} \boldsymbol{v}=2 \boldsymbol{w}_{0}-N_{0} C \boldsymbol{w}_{0}, \\
\boldsymbol{w}_{2}= & \left(2 I-N_{1} C\right) N_{1} \boldsymbol{v} \\
= & \left\{2 I-\left(2 I-N_{0} C\right) N_{0} C\right\} \boldsymbol{w}_{1} \\
= & 2 \boldsymbol{w}_{1}-\left(N_{0} C\right)\left\{2 \boldsymbol{w}_{1}-\left(N_{0} C\right) \boldsymbol{w}_{1}\right\}, \\
\boldsymbol{w}_{3}= & \left(2 I-N_{2} C\right) N_{2} \boldsymbol{v} \\
= & \left\{2 I-\left(2 I-N_{1} C\right) N_{1} C\right\} \boldsymbol{w}_{2} \\
= & {\left[2 I-\left\{2 I-\left(2 I-N_{0} C\right) N_{0} C\right\}\left(2 I-N_{0} C\right) N_{0} C\right] \boldsymbol{w}_{2} } \\
= & 2 \boldsymbol{w}_{2}-\left(N_{0} C\right)\left[4 \boldsymbol{w}_{2}-\left(N_{0} C\right) \times\right. \\
& \left.\quad\left\{6 \boldsymbol{w}_{2}-\left(N_{0} C\right)\left\{4 \boldsymbol{w}_{2}-\left(N_{0} C\right) \boldsymbol{w}_{2}\right\}\right\}\right] .
\end{aligned}
$$

When $l \geq 4$, it is possible to compute the vector $\boldsymbol{w}_{l}$ in the same manner as when $l \leq 3$. $\boldsymbol{w}_{l}$ requires only $N_{0} \boldsymbol{v}_{1}$ and $C \boldsymbol{v}_{2}$, and no matrix-matrix product is needed. In general, the number of matrix-vector products required by $\boldsymbol{w}_{l}$ is $2^{l}$. Therefore, $l$ should be relatively small in order to reduce the computation cost of the matrix-vector product. Compared with other preconditioners, fill-in must be considered in the incomplete $L U$ preconditioner, and the drop-off of nonzero entries is needed in the approximate inverse computed from the MR algorithm [8]. On the other hand, regarding the implicit implementation considered in this paper, the sparsity pattern of the preconditioner $N_{l}$ need not be considered since $N_{l}$ is not computed explicitly. This fact is also one of the advantages of computing $N_{l}$ implicitly. 


\section{Numerical experiments}

The numerical experiments are executed on a PC with Pentium4 $2.66 \mathrm{GHz}$ processor and $512 \mathrm{MB}$ main memory. In all examples, the Schur complement is generated from matrix $A$ to solve the reduced linear system (3).

\subsection{First example}

We discuss the boundary value problem of nonlinear partial differential equations in the region $\Omega=[0,1]^{3}$ (see Schönauer [5]):

$$
\left\{\begin{array}{c}
u_{x x}+u_{y y}+u_{z z}+D\left(u u_{x}+v u_{y}+w u_{z}\right)+u=f_{1} \quad \text { on } \Omega, \\
v_{x x}+v_{y y}+v_{z z}+D\left(u v_{x}+v v_{y}+w v_{z}\right)+v=f_{2} \quad \text { on } \Omega, \\
w_{x x}+w_{y y}+w_{z z}+D\left(u w_{x}+v w_{y}+w w_{z}\right)+w=f_{3} \quad \text { on } \Omega .
\end{array}\right.
$$

The right hand side functions $f_{1}, f_{2}$ and $f_{3}$, and the boundary condition are chosen so that the exact solutions are

$$
\begin{aligned}
u & =\sin (\pi x) \cos (\pi y) \cos (\pi z), \\
v & =\cos (\pi x) \sin (\pi y) \cos (\pi z), \\
w & =\cos (\pi x) \cos (\pi y) \sin (\pi z) .
\end{aligned}
$$

The problem (13) is discretized by the seven points central difference scheme using a square $40 \times 40 \times 40$ grid, where the mesh width $h$ is $1 / 41$. In the equation (13), note that $D$ is defined as the Reynolds number. In Table 1, $D$ is varied so that the product $D h$ is equal to $2^{-6}, 2^{-5}, 2^{-4}$ and $2^{-3}$. From the discretization, a nonlinear system of size 192,000 is generated. The discretized nonlinear system is usually solved by the following Newton scheme:

$$
\boldsymbol{q}_{l+1}=\boldsymbol{q}_{l}+J\left(\boldsymbol{q}_{l}\right)^{-1} \psi\left(\boldsymbol{q}_{l}\right),
$$

where $\boldsymbol{q}_{l}, \psi\left(\boldsymbol{q}_{l}\right)$ and $J\left(\boldsymbol{q}_{l}\right)$ are the $l$ th approximate solution, residual vector and Jacobi matrix of $\psi\left(\boldsymbol{q}_{l}\right)$, respectively. In the Newton formula (14), the 
linear system

$$
J\left(\boldsymbol{q}_{l}\right) \boldsymbol{x}=\psi\left(\boldsymbol{q}_{l}\right)
$$

must be solved once per iteration step. Although the discretized nonlinear system can also be solved by the inexact Newton scheme [9], it is not discussed in this paper. The linear system (15) is transformed into a reduced linear system with a Schur complement of size 128,000. In this example, when it is the first step of the Newton formula (14), the reduced linear system is solved by $\operatorname{GMRES}(m)$ algorithm [4]. The initial guess $\boldsymbol{q}_{0}$ is produced using the linear polynomial connecting two grid points: $(0, j h, k h)$ and $(1, j h, k h)$. We also apply the preconditioner $N_{l}$ to the reduced linear system using the implicit implementation, as mentioned in Subsection 3.2. The iteration of the $\operatorname{GMRES}(m)$ algorithm is terminated as soon as the stopping criterion on the residual norm,

$$
\left\|\boldsymbol{r}_{i}\right\|_{2} /\left\|\boldsymbol{r}_{0}\right\|_{2} \leq 1.0 \times 10^{-12},
$$

is satisfied, where $\boldsymbol{r}_{i}$ is the $i$ th residual vector of $\operatorname{GMRES}(m)$ algorithm. The zero vector is employed as the initial approximate solution of the reduced linear system. In the first experiment, the computation time of the initial guess $N_{0}$ is measured with respect to the cases of using the equations (7) and (8) when $D h=2^{-6}$. Even if the equation (7) is selected as the initial guess $N_{0}, N_{l} \boldsymbol{v}$ is not required explicitly for matrix $C^{T}$. Therefore, only the matrix norms $\|C\|_{1}$ and $\|C\|_{\infty}$ need to be computed. However, all the nonzero entries of matrix $C$ are required for obtaining $\|C\|_{1}$ and $\|C\|_{\infty}$, as mentioned in Subsection 3.1. On the other hand, if the equation (8) is selected, only the diagonal entries of $C$ are required.

Whereas the computation time of the matrix norms $\|C\|_{1}$ and $\|C\|_{\infty}$ is $5150.0 \mathrm{~s}$, that of the diagonal entries of $C$ is $1.9 \mathrm{~s}$. Therefore, the Newton formula (4) begins with the equation (8). Table 1 presents the computation time and iterations of the $\operatorname{GMRES}(m)$ algorithm that are required for satisfying the criterion (16). ' $N_{l}+\operatorname{GMRES}(m)$ ' and 'GMRES $(m)$ ' denote the $\operatorname{GMRES}(m)$ algorithm that is preconditioned using $N_{l}$ computed implicitly and the non-preconditioned $\operatorname{GMRES}(m)$ algorithm, respectively. The parameter $D h$ is varied from $2^{-6}$ to $2^{-3}$. In any restart of the $\operatorname{GMRES}(m)$ algorithm, 
TABLE 1: Example 4.1: The performance of the $\operatorname{GMRES}(m)$ algorithm (time: Computation time (s), iter: Iterations)

\begin{tabular}{|l|cc|cc|cc|cc|}
\hline \multirow{2}{*}{ Algorithm } & \multicolumn{10}{|c|}{$D h$} \\
\cline { 2 - 9 } & \multicolumn{2}{|c}{$2^{-6}$} & \multicolumn{2}{|c|}{$2^{-5}$} & $2^{-4}$ & $2^{-3}$ \\
\cline { 2 - 9 } & time & iter & time & iter & time & iter & time & iter \\
\hline GMRES(20) & 116.0 & 415 & 122.0 & 437 & 127.0 & 453 & 132.0 & 474 \\
$N_{0}+$ GMRES(20) & 117.0 & 414 & 121.0 & 432 & 123.0 & 440 & 134.0 & 474 \\
$N_{1}+$ GMRES(20) & 60.0 & 163 & 56.0 & 152 & 54.0 & 147 & 62.0 & 170 \\
$N_{2}+$ GMRES(20) & 54.0 & 101 & 55.0 & 105 & 55.0 & 104 & 57.0 & 108 \\
$N_{3}+$ GMRES(20) & 54.0 & 61 & 55.0 & 64 & 60.0 & 69 & 63.0 & 73 \\
\hline GMRES(30) & 107.0 & 339 & 101.0 & 330 & 112.0 & 355 & 125.0 & 395 \\
$N_{0}+$ GMRES(30) & 102.0 & 326 & 104.0 & 330 & 112.0 & 355 & 122.0 & 389 \\
$N_{1}+$ GMRES(30) & 55.0 & 139 & 55.0 & 139 & 55.0 & 139 & 54.0 & 137 \\
$N_{2}+$ GMRES(30) & 45.0 & 80 & 49.0 & 87 & 54.0 & 96 & 55.0 & 100 \\
$N_{3}+$ GMRES(30) & 54.0 & 59 & 54.0 & 60 & 56.0 & 62 & 58.0 & 64 \\
\hline GMRES(40) & 115.0 & 329 & 117.0 & 335 & 105.0 & 302 & 125.0 & 354 \\
$N_{0}+$ GMRES(40) & 107.0 & 308 & 100.0 & 287 & 104.0 & 300 & 120.0 & 347 \\
$N_{1}+$ GMRES(40) & 53.0 & 122 & 54.0 & 124 & 59.0 & 138 & 62.0 & 145 \\
$N_{2}+$ GMRES(40) & 50.0 & 85 & 52.0 & 87 & 53.0 & 90 & 55.0 & 93 \\
$N_{3}+$ GMRES(40) & 51.0 & 56 & 54.0 & 58 & 57.0 & 62 & 60.0 & 65 \\
\hline
\end{tabular}




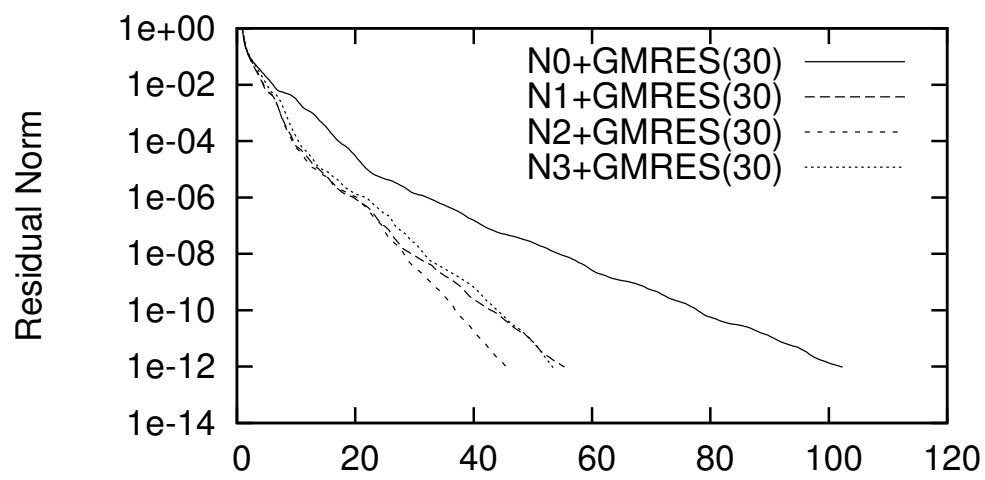

(a)

Computation Time (s)

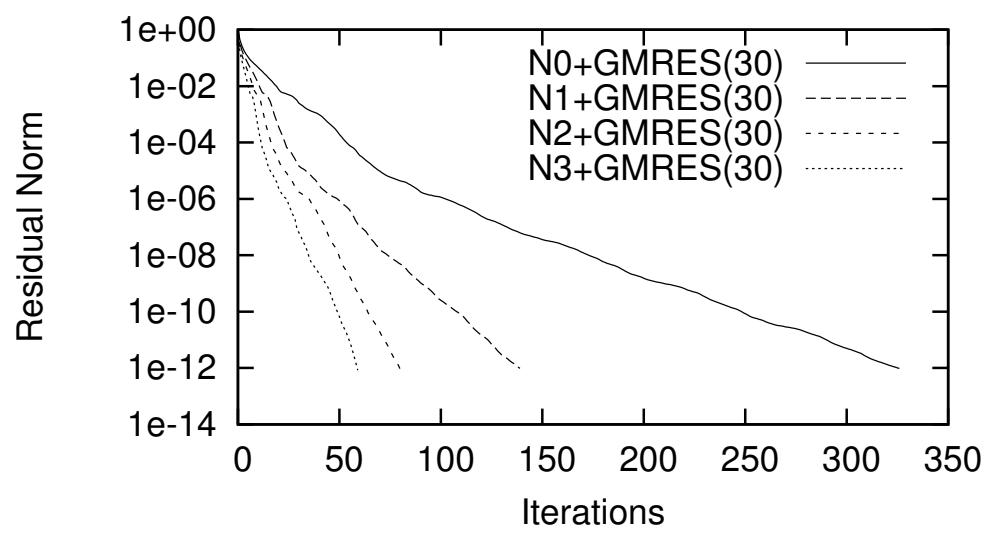

(b)

Figure 1: Example 4.1: The convergence behavior of the preconditioned GMRES(30) algorithm, $\left(D h=2^{-6}\right)$ : (a) Residual norm versus Time (s); (b) Residual norm versus Iterations. 
the $N_{0}+\operatorname{GmRES}(m)$ and $\operatorname{GmReS}(m)$ algorithms do not converge within $100.0 \mathrm{~s}$. On the other hand, the $N_{1}+\operatorname{GmRes}(m), N_{2}+\operatorname{GMREs}(m)$ and $N_{3}+\operatorname{GMRES}(m)$ algorithms converge in 45.0 to $77.0 \mathrm{~s}$. Figure 1 illustrates the behavior of the residual norm of the preconditioned GMRES(30) algorithm when $D h=2^{-6}$. While the iterations of the $N_{2}+$ GMRES(30) algorithm cost more than those of the $N_{3}+\operatorname{GmRES}(30)$ algorithm, the computation time of the former is less expensive than that of the latter. Also the convergence behaviors of the $N_{1}+\operatorname{GmRES}(30)$ and the $N_{3}+\operatorname{GmRES}(30)$ algorithms are almost the same. Moreover, the computation cost of $N_{l}$ in explicit form is measured in order to compare it with $N_{l} \boldsymbol{v}$ when $l=1,2,3$. The computation time of $N_{1}$ in explicit form is $5383.0 \mathrm{~s}$. Obviously, the explicit computation of $N_{1}$ is more expensive than the computation of the $\operatorname{GMRES}(m)$ algorithm preconditioned implicitly. It is impossible to compute $N_{2}$ and $N_{3}$ explicitly due to insufficient strategies. Therefore, the explicit computation of $N_{l}$ is not effective. The time of the convergence of all the GMRES $(m)$ algorithms preconditioned with the equation (8) in Table 1 is less than the time required for obtaining $N_{0}$ from the equation (7), because the computation time of $\|C\|_{1}$ and $\|C\|_{\infty}$ is $5150.0 \mathrm{~s}$. The computation of $N_{0}$ with the equation (8) is included in the computation time in Table 1. Therefore, it is more effective to select the equation (8) instead of the equation (7) in Example 4.1. From these reasons, we do not discuss the convergence of the $\operatorname{GMRES}(m)$ algorithm that is preconditioned using the equation (7).

\subsection{Second example}

In this example, we discuss three types of linear systems with coefficient matrices in Florida Sparse Matrix Collection [10]. Their coefficient matrices are termed 'EPB3', 'LUNG2' and 'TORSO2'. The size and nonzero entries of these three matrices are listed in Table 2. For more details on these matrices, see [10]. In all linear systems, the right hand side is determined so that all the entries of the exact solution are 1.0. Similar to Example 4.1, a reduced linear system is produced and solved by the $\operatorname{GMRES}(m)$ algorithm, where the size 
TABLE 2: Example 4.2: The characteristics of the coefficient matrices

\begin{tabular}{|l|ccc|}
\hline Matrix name & Size & Nonzero entries & Size of Schur complement \\
\hline EPB3 & 84617 & 463625 & 63318 \\
LUNG2 & 109460 & 492564 & 82104 \\
TORSO2 & 115967 & 1033473 & 86658 \\
\hline
\end{tabular}

of the Schur complement is given in Table 2. In this example, the computation time of the initial guess $N_{0}$ is measured. Regarding 'EPB3', 'LUNG2' and 'TORSO2', the computation times of the diagonal entries of matrix $C$ are 0.26 , 0.31 and $0.43 \mathrm{~s}$, respectively. On the other hand, the computation times of the matrix norms $\|C\|_{1}$ and $\|C\|_{\infty}$ are 1089.0, 1729.0 and 3097.0 s, respectively. Therefore, we select the equation (8) as the initial guess $N_{0}$. Table 3 shows the computation time and the iterations of the $\operatorname{GmREs}(m)$ algorithm. The preconditioner $N_{l}$ is computed implicitly using the matrix-vector product. In all linear systems, the preconditioned $\operatorname{GMRES}(m)$ algorithm works better than the non-preconditioned $\operatorname{GMRES}(m)$ algorithm. Particularly in the case of 'EPB3', the preconditioner $N_{l}$ makes the convergence faster as $l$ is increased. Moreover, the computation time of the preconditioner $N_{l}$ in explicit form is measured. With regard to 'EPB', 'TORSO2' and 'LUNG2', the computation times of $N_{1}$ are $936.0,1504.0$ and $2659.0 \mathrm{~s}$, respectively. In all these cases, preconditioners $N_{2}$ and $N_{3}$ cannot be computed since the strategies are inadequate. Therefore, computing $N_{l}$ explicitly is more expensive than computing it implicitly using the matrix-vector product. In Table 3 , some of $\operatorname{GMRES}(m)$ algorithms that are preconditioned with $N_{l}$ using the equation (8) converge for 'EPB'. Regarding the case of 'EPB', we compute $N_{1}, N_{2}$ and $N_{3}$ implicitly based on the equation (7) and apply it to the GMRES $(m)$ algorithm for comparison with the equation (8). However, in any restart of the GM$\operatorname{RES}(m)$ algorithms that are preconditioned with $N_{1}, N_{2}$ and $N_{3}$ using the equation (7), the residual norm could not converge within $15 \mathrm{~min}$. Therefore, the equation (8) is a better selection than the equation (7). It is also 
TABLE 3: Example 4.2: The performance of the $\operatorname{GMRES}(m)$ algorithm (time: Computation time (s), iter: Iterations)

\begin{tabular}{|l|cc|cc|cc|}
\hline \multirow{2}{*}{ Algorithm } & \multicolumn{6}{|c|}{ Matrix name } \\
\cline { 2 - 7 } & \multicolumn{2}{|c|}{ EPB3 } & \multicolumn{2}{c|}{ LUNG2 } & \multicolumn{2}{c|}{ TORSO2 } \\
\cline { 2 - 7 } & time & iter & time & iter & time & iter \\
\hline GMRES(20) & - & - & - & - & 8.0 & 66 \\
$N_{0}+$ GMRES(20) & - & - & 120.0 & 1305 & 5.0 & 35 \\
$N_{1}+$ GMRES(20) & - & - & 57.0 & 536 & 3.0 & 20 \\
$N_{2}+$ GMRES(20) & - & - & 43.0 & 301 & 3.0 & 13 \\
$N_{3}+$ GMRES(20) & 185.0 & 1063 & 42.0 & 190 & 3.0 & 9 \\
\hline GMRES(30) & - & - & - & - & 9.0 & 64 \\
$N_{0}+$ GMRES(30) & - & - & 121.0 & 1161 & 5.0 & 35 \\
$N_{1}+$ GMRES(30) & - & - & 57.0 & 457 & 3.0 & 20 \\
$N_{2}+$ GMRES(30) & 445.0 & 3514 & 43.0 & 263 & 3.0 & 13 \\
$N_{3}+$ GMRES(30) & 226.0 & 1203 & 41.0 & 168 & 3.0 & 9 \\
\hline GMRES(40) & - & - & - & - & 9.0 & 65 \\
$N_{0}+$ GMRES(40) & - & - & 132.0 & 1070 & 5.0 & 34 \\
$N_{1}+$ GMRES(40) & 674.0 & 6084 & 60.0 & 424 & 3.0 & 20 \\
$N_{2}+$ GMRES(40) & 278.0 & 1962 & 44.0 & 244 & 3.0 & 13 \\
$N_{3}+$ GMRES(40) & 195.0 & 963 & 41.0 & 159 & 3.0 & 9 \\
\hline
\end{tabular}

$(-)$ : the algorithm could not converge within $15 \mathrm{~min}$. 
suggested that the good approximate preconditioner is not obtained within three iterations of the Newton scheme even if the equation (7) is selected as the initial guess.

\section{Concluding remarks}

We implemented the preconditioner $N_{l}$ of the reduced linear system implicitly by using the matrix-vector product. By implementing it implicitly, no matrix-matrix product is needed. Moreover, we also selected the diagonal matrix as the initial guess of the Newton iteration in order to make it easy to compute. The numerical results show that the computation of $N_{1}$ costs more than that of $N_{1} \boldsymbol{v}$. The preconditioner $N_{l}$ in explicit form cannot be obtained if $l \geq 2$. Therefore, the implicit implementation considered in this paper is a better alternative to computing the preconditioner $N_{l}$ explicitly.

\section{References}

[1] G. Shulz. Iterative Berechnung der Reziproken Matrix, Z. Angew. Math. Mech., 13: 57-59, 1933. C397

[2] A. S. Householder. The Theory of Matrices in Numerical Analysis, Dover, New York, 1964. C398

[3] A. Ben-Israel and D. Cohen. On Iterative Computation of Generalized Inverses and Associated Projections, SIAM J. Numer. Anal., 3: 410-419, 1966. C397

[4] Y. Saad and M. H. Schultz. GMRES: A Generalized Minimal Residual Algorithm for Solving Nonsymmetric Linear Systems, SIAM J. Sci. Stat. Comput., 7: 856-869, 1986. C401 
[5] W. Schönauer. Scientific Computing on Vector Computers, North Holland, 1987. C400

[6] V. Pan and R. Schreiber. An Improved Newton Iteration for the Generalized Inverse of a Matrix with Applications, SIAM J. Sci. Stat. Comp., 12(5): 1109-1130, 1991. C398

[7] Y. Saad. Iterative Methods for Sparse Linear Systems, PWS Publishing Company, Boston, 1996. C396

[8] T. Huckel. Approximate Sparsity Patterns for the Inverse of a Matrix and Preconditioning, Appl. Numer. Math., 30: 291-303, 1999. C397, C399

[9] X. C. Cai and D. E. Keyes. Nonlinearly Preconditioned Inexact Newton Algorithms, SIAM J. Sci. Comput., 41(1): 183-200, 2002. C401

[10] University of Florida Sparse Matrix Collection. [Online] http://www.cise.ufl.edu/research/sparse/matrices. C404 